\title{
AE Frequency analysis of Damage Mechanism in CFRP Laminates Based on Hilbert-Huang Transform
}

\author{
Wen-Qin HAN ${ }^{1, a^{*}}$ and Ying LUO ${ }^{1, b}$, \\ ${ }^{1}$ Faculty of Civil Engineering and Mechanics, Jiangsu University, Jiangsu Province,Zhenjiang, China \\ ahwq402@163.com, 'luoying@mail.ujs.edu.cn \\ ${ }^{*}$ Corresponding author
}

Keywords: Composite, Acoustic Emission, Peak Frequency, Hilbert-Huang Transform.

\begin{abstract}
To gain a deeper understanding of damage evolution in carbon fiber-reinforced plastic (C FRP) composite materials, This paper addresses an application of recently developed Hilbert-Huang transform (HHT) signal processing technique on acoustic emission (AE) signals feature extraction of damage Mechanism in CFRP laminates. The composite structures for lay-ups and orientations are becoming more and more complicated, a AE signal is likely to include more variety of components produced by various damage modes simultaneously, the AE signal were decomposed by HHT metho d, Fast Fourier Transform (FFT) was performed for the intrinsic mode functions (IMF) components, the peak frequency of each IMF component was used as a new descriptor for identifying damage mo des. So HHT can provide promising methods for frequency features extraction of all damage modes and be a better tool compared to FFT to help understanding the damage process.
\end{abstract}

\section{Introduction}

Fiber-reinforced plastic (FRP) composite materials are exten-sively used in various engineering applications because of their high specific stiffness and strength. However, various damage mechanisms may degrade the long-term performance of these materials, it is necessary to study damage mode and mechanism of composite. Composite structure failure often manifest as macro-damage gene rated by a few accumulative meso-damages along with time, which include matrix cracking, fiber breakage, delamination, and debonding. When each meso-damage happens, acoustic emission phenomenon will take place, and obvious AE signal feature corresponds to each meso-damage [1]. Hence, discrimination of $\mathrm{AE}$ signals corresponding to different failure mechanisms is of great im-portance in the use of AE technique.

Various signal processing and pattern recognition techniques have been performed for damage feature extraction from AE signals [2-9], which have four main methods such as: (1) damage classification according to a single AE parameter (amplitude, frequency, etc.), (2) wavelet level, (3) pattern recognition using several AE parameters,(4) classification according to the extensional and flexural mode content.

The objective of the current work is to identify characteristic signal features associated with damage propagation for each failure mode on CFRP laminate. Time-frequency analysis of AE signals was performed using Empirical Mode Decomposi-tion. AE signals were decomposed into a set of Intrinsic Mode Functions components. AE signals were post-processed using Hilbert-Huang Transform. The event peak frequency of each IMF component was obtained from the classical Fast Fourier Transform of each IMF component waveform. It was shown that the event peak frequency could be directly related to the materials damage modes respectively.

\section{Experiment Procedure}

Standard tensile test were carried out, the tensile specimen is an unidirectional ply specimen with two slot (see Fig.1), which were made of a lay-up of an unidirectional carbon/epoxy pre-preg sheets. The AE sensors used were broadband (WD) sensors with a operating frequency range of $25-750 \mathrm{kHz}$ and the signals were pre-amplified using a 20/40/60 db preamplifier, the gain selector of the preamplifier 
was set to $40 \mathrm{~dB}$. Grease was used for gaining good acoustic coupling on the surface of the sensor. A threshold of $40 \mathrm{~dB}$ was adopted considering the environmental noise, the mechanical and electrical noises detected with signals having durations below $20 \mu$ s were all screened in the AE parametric analysis.

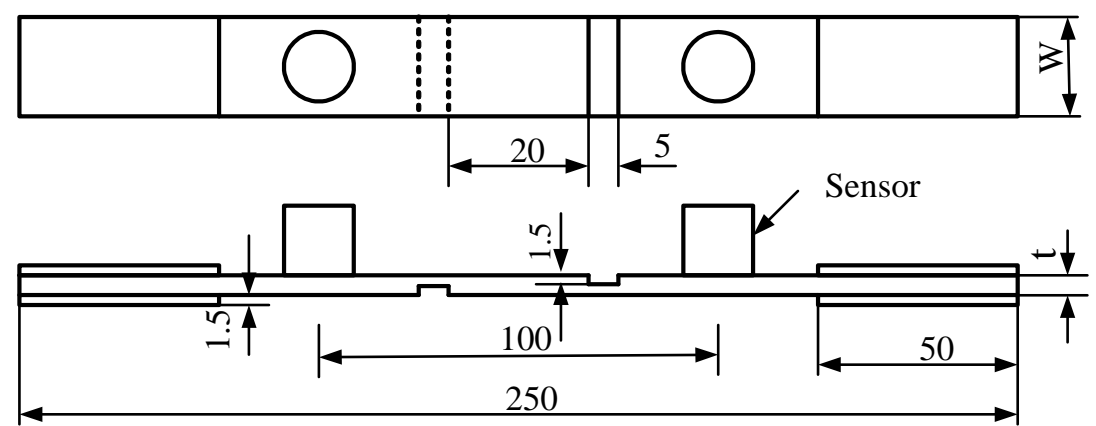

Fig. 1 The geometries and sensor locations for specimen

\section{Hilbert-Huang Transform}

Hilbert Huang Transform (HHT) is a data analysis tool which is able to extract the cyclic components from a signal. It has the capability dealing with non-stationary and nonlinear data. The HHT technique for analyzing data consists of two components: a decomposition algorithm called Empirical Mode Decomposition (EMD) and a spectral analysis tool called Hilbert spectral analysis.EMD is an algorithm which decomposes an input signal into a finite set of oscillating functions which are the intrinsic cycles of the original signal (see Eq.1). Hilbert spectral analysis is a tool which calculates and displays the amplitude or energy (square of amplitude) contributions from the different extracted cyclic components as a function of time (see Eq.2). Using these tools, the internal cycles of a signal, whether nonstationary or nonlinear, can be displayed and analyzed in the time domain or as time - frequency - energy spectra.

$$
\begin{aligned}
& x(t)=\sum_{j=1}^{n} c_{j}(t)+r_{n}(t) . \\
& H(\omega, t)=\sum_{i=1}^{n} A_{i}(t)
\end{aligned}
$$

\section{Result and Analysis}

Fig. 2 shows the evolvement of load and amplitude and peak frequency of AE signal versus time for specimen respectively. It is obvious that the whole tensile testing was distributed into three stages from Fig. 2. In the first stage (0-100 s), the AE amplitude value is small, the main range of peak frequency is only $140-190 \mathrm{kHz}$. In the second stage (100-230 s), the AE signals of the high-amplitude increase remarkably, the AE signals about the ranges of $20-50 \mathrm{kHz}$ and $230-290 \mathrm{kHz}$ begin to produce, the load fluctuate and is in the same value due to the rapid crack propagation parallel with the loading direction, which was visually inspected by naked eye. During the second stage , delamination take place clearly, which leads to increase fibre/matrix interface debonding and fiber pull-out. In addition to these identified failure modes, contact and friction on the fracture surfaces can also contribute to the generation of AE signals. In the third stage (230-300 s), the AE amplitude value become lower, the AE signals on the frequency range of $350-450 \mathrm{kHz}$ increase gradually, which corresponds to fiber breakage. It is seen that the peak frequency range may be divided into five frequency bands, that is to say, there are several kinds of damage modes in the tensile test. 

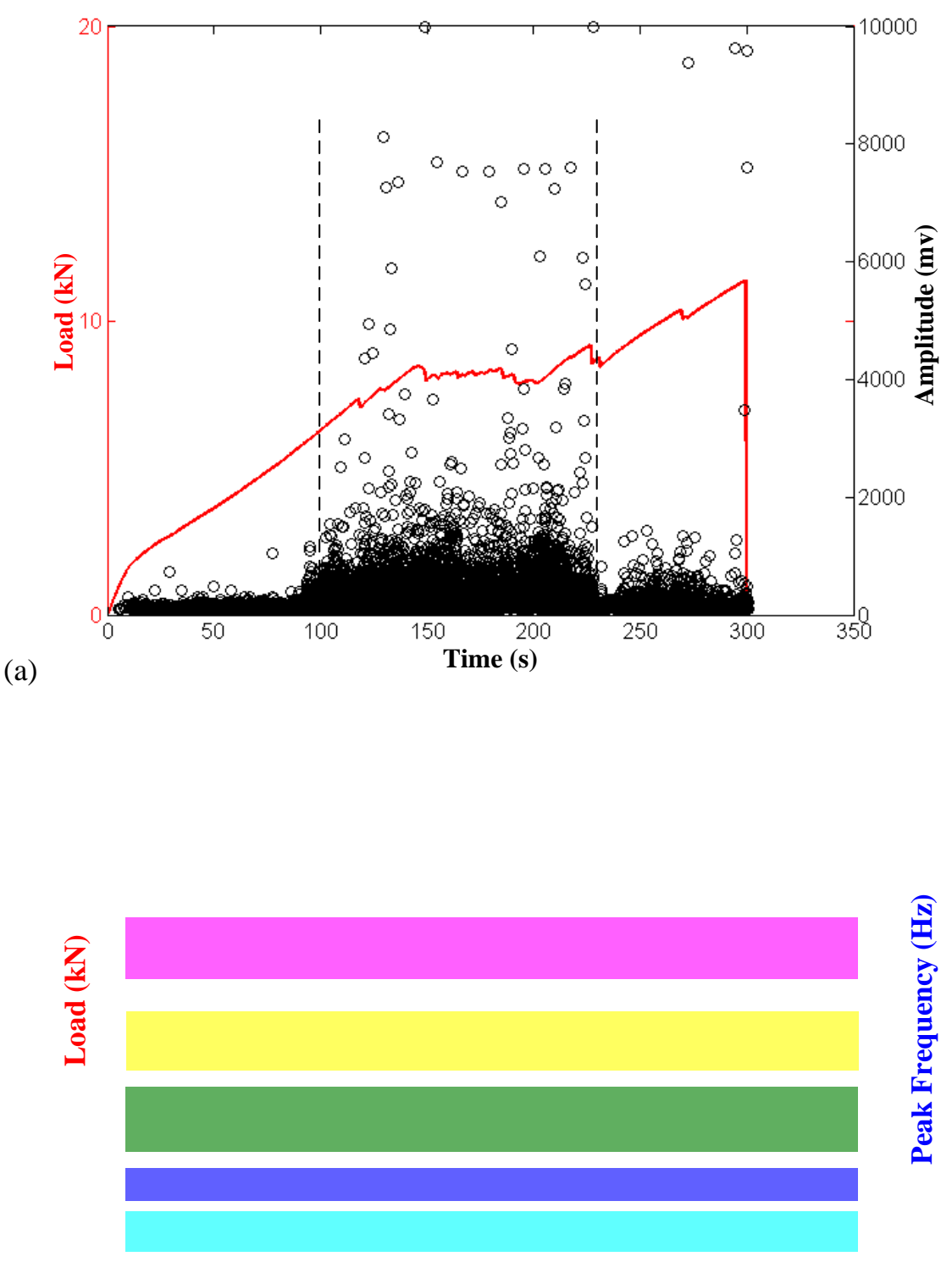

(b)

Time (s)

Fig. 2. The AE evolvement of Specimen: (a) amplitude and load versus time;(b) peak frequency and load versus time

A AE signal was chosen to perform FFT and HHT analysis at $295.291 \mathrm{~s}$, the waveform and FFT of the AE signal are shown in Fig. 3. It is seen that the FFT power spectrum in this specific case shows several peaks(see Fig.3(b)), but the peak frequency of the AE signal is $169 \mathrm{kHz}$. A possible interpretation is that the waveform is formed from several individual sources associated with several damage modes and each one has its own frequency. In order to distinguish the damage modes included in the AE signal, which was decomposed into several IMF components, the FFT analysis was performed on every IMF waveform, 1-4 IMF waveforms and FFT of the AE signal are shown in Fig. 4. Because the decomposed IMF components were stable signal sequences, the peak frequency was chosen to act as the dominant frequency of IMF waveform, which is associated with specific damage mode. It is seen that the dominant frequency of each IMF waveform is $169,111,57 \mathrm{kHz}$ from high to low respectively, the dominant frequency possibly corresponds to different damage modes of composites. In addition, it is obvious that there are two peak values in FFT of IMF1, which consist of $169 \mathrm{kHz}$ and $399 \mathrm{kHz}$. The waveform of IMF1 is divided into two parts, the front and end part correspond to $399 \mathrm{kHz}$ and $169 \mathrm{kHz}$ respectively, so, the AE signal consists of four kinds of the 
dominant frequency. The three-dimensional united time-frequency chart of the AE signal are shown in Fig. 3(c), it is seen that the AE signal includes a some kinds of signal components, there are main frequency ranges of $320-400 \mathrm{kHz}$ and $140-190 \mathrm{kHz}, 80-120 \mathrm{kHz}$, as well as $30-70 \mathrm{kHz}$, which is the lower amplitude signal.
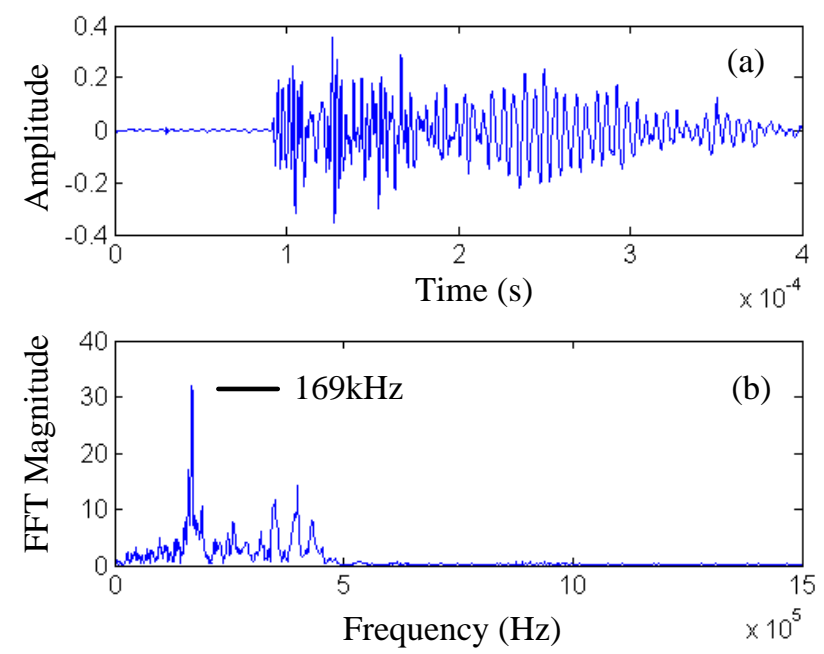

(a)

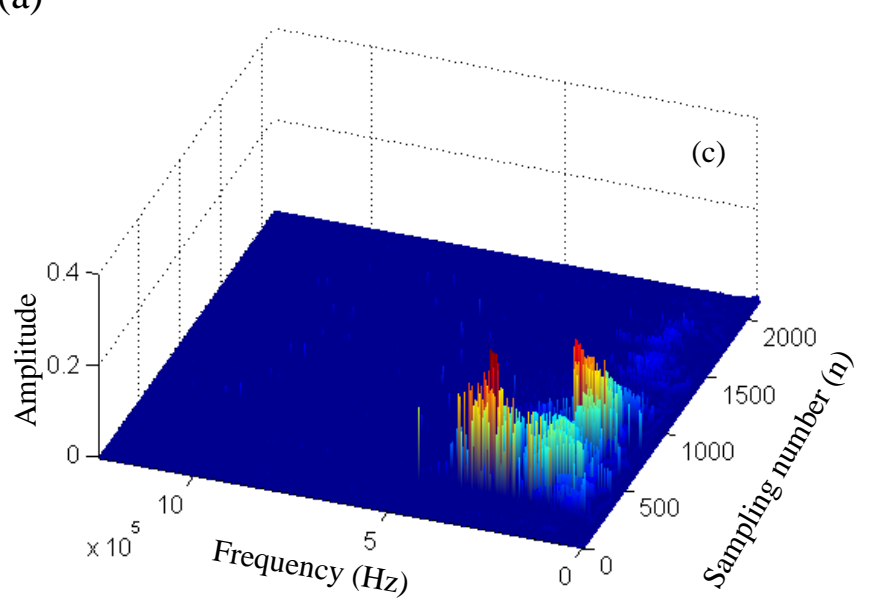

(b)

Fig. 3. (a) The AE waveform at 295.291 s; (b) frequecy domain;(c)The three-dimensional united time-frequency chart
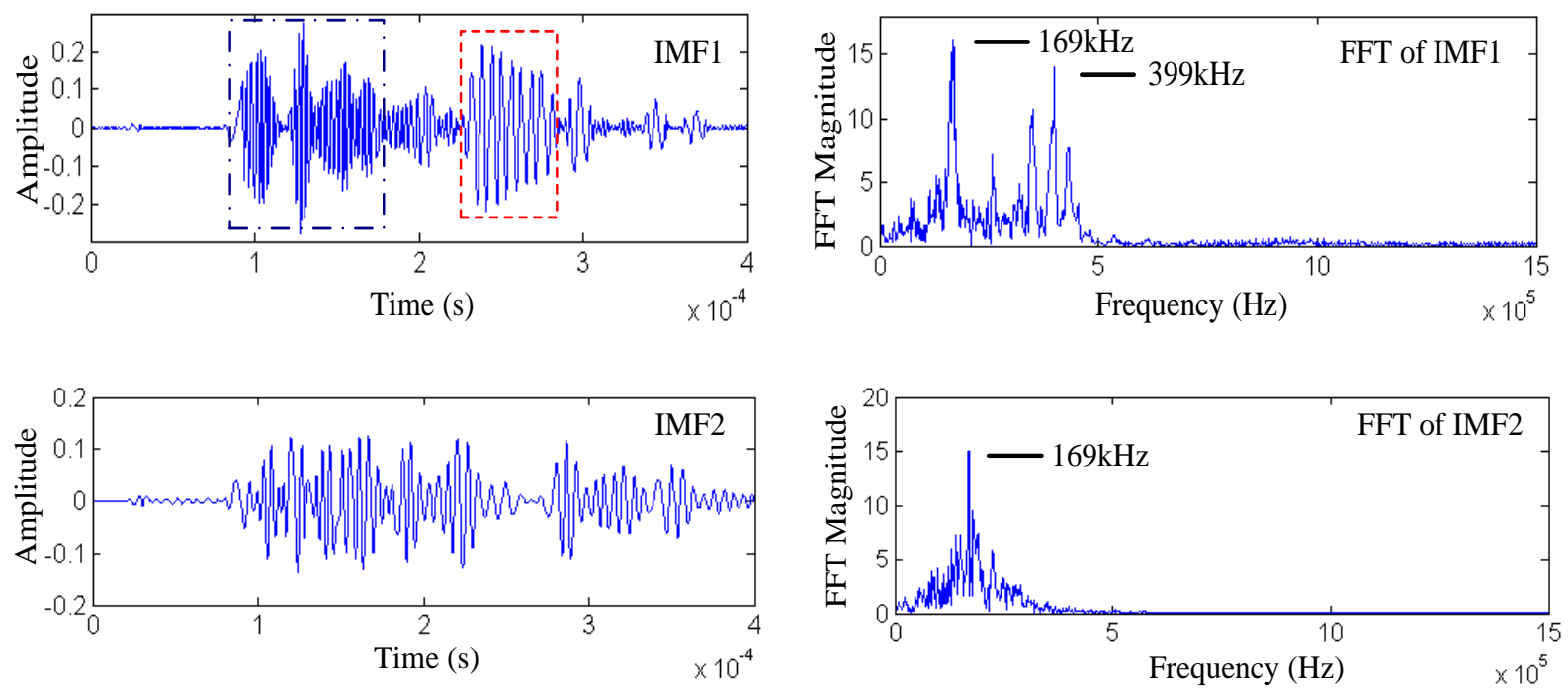

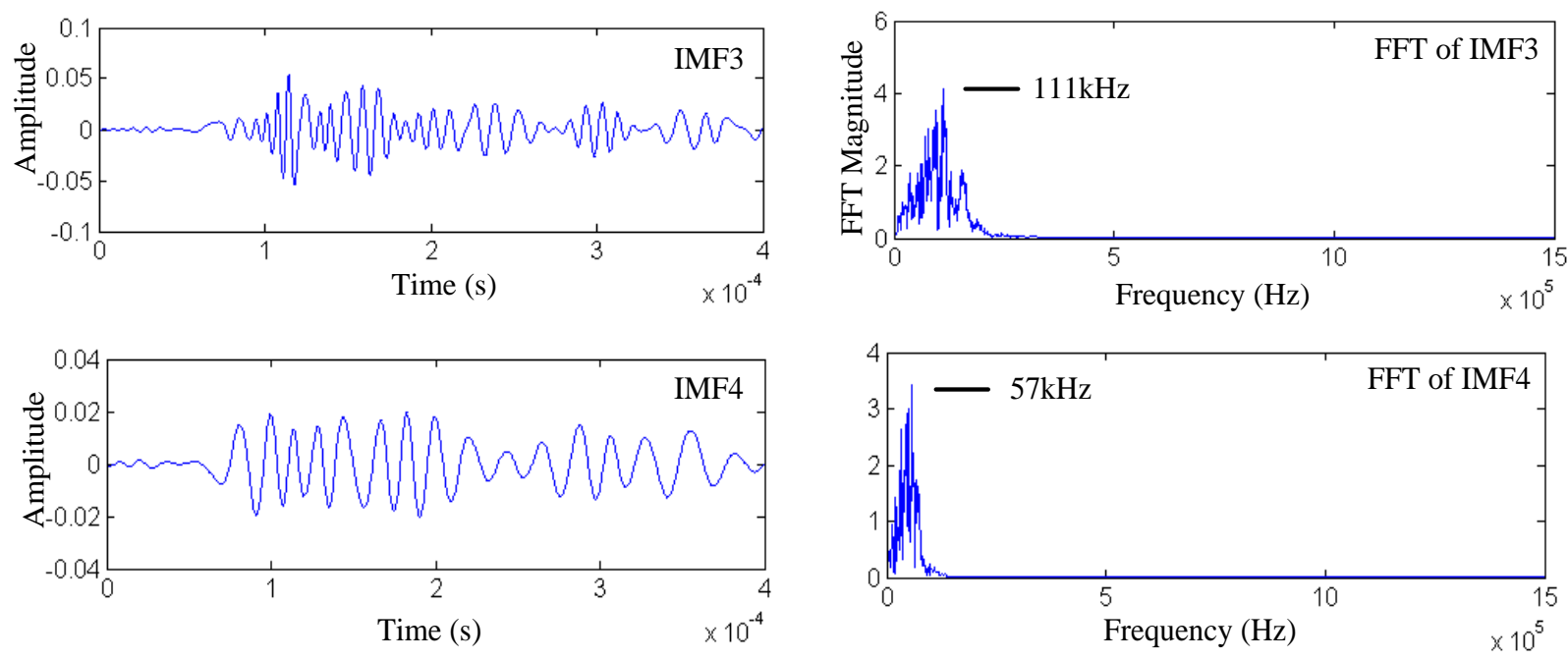

Fig. 4. EMD of the AE signal at 295.291 s and FFT of each IMF.

\section{Summary}

The damage mode corresponding to the peak frequency of an AE signal is just the principal damage component in this AE signal possibly, therefore, it is necessary to detach AE signal and extract all damage modes existing in a AE signal by EMD and HHT. AE signal was decomposed into several IMF components by means of EMD algorithm, and the FFT analysis was performed on every IMF waveform, the analysis results show that the various damages can be detached and extracted effectively, and the peak frequency of each IMF was chosen to act as a new descriptor for identifying damage modes contained within an AE signal. Hilbert spectrum obtained by HHT can described the change rule of the amplitude along with frequency and time, the frequency distribution of the IMF component in time-scale is clearly expressed on the three-dimensional united time-frequency chart of a AE signal, which is easy to distinguish the frequency content of an AE signal.

In summary, characterisation of failure modes in CFRP composite using the peak frequency of AE signal appears to be an effective method, but the composite structures for lay-ups and orientations are becoming more and more complicated, a AE signal include more variety of components produced by various damage modes simultaneously, the HHT provide promising methods for non-stationary AE signals features extraction.

\section{Acknowledgement}

This work was supported by the National Natural Science Foundation of China (Grant No.11272138) and the Specialized Research Fund for the Doctoral Program of Higher Education (Grant

No.20123227130002).

\section{References}

[1] Peng F, Fu Y M, Chen Y J, Effect of matrix cracking on the time delayed buckling of viscoelastic laminated circular cylindrical shells. Sci China Tech Sci 51 (2008) 2152-2164

[2] Dickinson LP, Fletcher NH, Acoustic detection of invisible damage in aircraft composite panels. Appl Acoust 70 (2009) 110-119

[3] Xingmin Z, Xiong Y, Investigation of damage mechanisms in self-reinforced polyethylene composites by acoustic emission. Compos Sci Technol 66 (2006) 444-449 
[4] Ni Q, Iwamoto M, Wavelet transform of acoustic emission signals in failure of model composites. Eng Fract Mech 69 (2002) 717-728

[5] Godina N, Hugueta S, Gaertnera R, et al, Clustering of acoustic emission signals collected during tensile tests on unidirectional glass/ polyester composite using supervised and unsupervised classifiers. NDT\&E Int 37 (2004) 253-264

[6] S.E. Hamdi, A.L. Duff, L.Simon, et al, Acoustic emission pattern recognition approach based on Hilbert-Huang transform for structural health monitoring in polymer-composite materials. Appl Acoust 74 (2013) 746-757

[7] José Martínez-Jequier, Antolino Gallego, Elisabet Suárez, et al, Real-time damage mechanisms assessment in CFRP samples via acoustic emission Lamb wave modal analysis. Composites: Part B 68 (2015) 317-326

[8] Safaa Kh. Al-Jumaili, Karen M. Holford, Mark J. Eaton, et al, Parameter Correction Technique (PCT): A novel method for acoustic emission characterisati on in large-scale composites. Composites Part B 75 (2015) 336-344

[9] V. Munoz, B. Vales, M. Perrin, et al, Damage detection in CF RP by coupling acoustic emission and infrared thermography. Composites Part B 85 (2016) 68 -75 